\title{
Combination of quasiconvex subgroups of relatively hyperbolic groups
}

\author{
Eduardo Martínez-Pedroza
}

\begin{abstract}
For relatively hyperbolic groups, we investigate conditions guaranteeing that the subgroup generated by two quasiconvex subgroups $Q_{1}$ and $Q_{2}$ is quasiconvex and isomorphic to $Q_{1} * Q_{1} \cap Q_{2} Q_{2}$. Our results generalize known combination theorems for quasiconvex subgroups of word hyperbolic groups. Some applications are presented.
\end{abstract}

Mathematics Subject Classification (2000). 20F65, 20F67.

Keywords. Relatively hyperbolic group, quasi-convex subgroup, combination theorem, parabolic subgroup.

\section{Introduction}

Relatively hyperbolic groups, originally introduced by M. Gromov [18], have received a great deal of attention by group theorists after foundational works by B. Farb [16] and B. H. Bowditch [9] in the late 1990s. This class of groups includes many interesting subclasses - for instance, limit groups which are an essential part of the theory of algebraic geometry over free groups [2], [14], and geometrically finite Kleinian groups which contain fundamental groups of finite-volume hyperbolic manifolds.

If $G$ is a countable group and $\mathscr{H}$ is a collection of subgroups of $G$, the notion of relative hyperbolicity for the pair $(G, \mathscr{H})$ has been defined by different authors [9], [15], [16], [18], [19], [20], [25], [30]. All these definitions are equivalent when the group $G$ and the subgroups in $\mathscr{H}$ are finitely generated [15], [18], [25], [29], [30]. A precise definition is provided in the next section. When a pair $(G, \mathscr{H})$ satisfies the relative hyperbolicity condition we say that the group $G$ is hyperbolic relative to $\mathscr{H}$, and when the collection $\mathscr{H}$ is fixed we just say that the group $G$ is relatively hyperbolic.

For a group $G$ hyperbolic relative to a collection of subgroups $\mathscr{H}$, the quasiconvex subgroups are the natural subgroups to study when considering a relatively hyperbolic group as a geometric object. Different notions of relative quasiconvexity for subgroups 
of $G$ were introduced by F. Dahmani [14] and D. Osin [25], and recently C. Hruska has proved the equivalence of these definitions [20].

We are interested in the following problem.

Problem 1. Let $G$ be a relatively hyperbolic group, and suppose that $Q$ and $R$ are quasiconvex subgroups of $G$. Consider the natural homomorphism

$$
\rho: Q * Q \cap R R \rightarrow G,
$$

which has image the subgroup $\langle Q \cup R\rangle$.

(1) (Algebraic structure) When is $\rho$ injective?

(2) (Geometric structure) When is the image of $\rho$ a quasiconvex subgroup?

1.1. Main results. Let $G$ be a group generated by a finite set $X$ and hyperbolic relative to a collection of subgroups $\mathscr{H}$. A subgroup of $G$ is called parabolic if it can be conjugated into one of the subgroups in $\mathscr{H}$. For an element $g \in G,|g|_{X}$ denotes its distance from the identity element in the word metric induced by $X$ on $G$.

Theorem 1.1 (Quasiconvex-parabolic amalgamation). For any relatively quasiconvex subgroup $Q$ and any maximal parabolic subgroup $P$ of $G$, there is a constant $C=C(Q, P) \geq 0$ with the following property. If $R$ is a subgroup of $P$ such that

(1) $Q \cap P \leq R$, and

(2) $|g|_{X} \geq C$ for any $g \in R \backslash Q$,

then the natural homomorphism

$$
Q * Q \cap R R \rightarrow G
$$

is injective with image a relatively quasiconvex subgroup.

Moreover, every parabolic subgroup of $\langle Q \cup R\rangle<G$ is either conjugate to a subgroup of $Q$ or a subgroup of $R$ in $\langle Q \cup R\rangle$.

Theorem 1.2 (Quasiconvex-quasiconvex amalgamation). For any pair of relatively quasiconvex subgroups $Q_{1}$ and $Q_{2}$, and any maximal parabolic subgroup $P$ such that $R=Q_{1} \cap P=Q_{2} \cap P$, there is a constant $C=C\left(Q_{1}, Q_{2}, P\right) \geq 0$ with the following property. If $h \in P$ is such that

(1) $h R h^{-1}=R$, and

(2) $|g|_{X} \geq C$ for any $g \in R h R$, then the natural homomorphism

$$
Q_{1} *_{R} h Q_{2} h^{-1} \rightarrow G
$$

is injective and its image is a relatively quasiconvex subgroup. 
Moreover, every parabolic subgroup of $\left\langle Q_{1} \cup h Q_{2} h^{-1}\right\rangle\langle G$ is either conjugate to a subgroup of $Q_{1}$ or $h Q_{2} h^{-1}$ in $\left\langle Q_{1} \cup h Q_{2} h^{-1}\right\rangle$.

1.2. History and motivation. This work is motivated by known combination theorems for quasiconvex subgroups of word hyperbolic groups. In [18], M. Gromov stated that in a torsion-free word hyperbolic group any infinite index quasiconvex subgroup is a free factor of a larger quasiconvex subgroup. Gromov's ideas were developed by G. N. Arzhantseva in [4]. More general combination theorems for quasiconvex subgroups of word hyperbolic groups were stated and proved by R. Gitik in [17].

For relatively hyperbolic groups, G. Arzhantseva and A. Minasyan use a combination theorem for cyclic subgroups to prove that relatively hyperbolic groups which do not have non-trivial finite normal subgroups are $C^{*}$-simple [5]. In the case of relatively hyperbolic groups with discrete representations in $\operatorname{Isom}\left(\mathbb{H}^{n}\right)$, results by M. Baker and D. Cooper correspond to the combination of quasiconvex subgroups [6].

The Klein-Maskit Combination Theorems for Kleinian groups [23] are another motivation for our work; in particular, the following example whose details can be found in [21]: if $G_{1}$ and $G_{2}$ are two lattices of $\operatorname{PSL}(2, \mathbb{C})$ and $R$ is a maximal parabolic subgroup of both, then for a "sufficiently complicated" parabolic $h$ centralizing $R$, the natural homomorphism from $Q_{1} *_{R} h Q_{2} h^{-1}$ into $\operatorname{PSL}(2, \mathbb{C})$ is injective. This technique has been used by D. Cooper, D. Long, and A. Reid to double quasi-Fuchsian subgroups along parabolic subgroups in hyperbolic manifold groups, producing essential closed surfaces in cusped hyperbolic manifolds [13], [12]. Corollary 1.7 illustrates this technique in the context of relatively hyperbolic groups.

Another motivating result, with a similar statement to Theorem 1.2, is a combination theorem for Veech subgroups of the mapping class group by $\mathrm{C}$. Leininger and A. Reid in [21]. This result was used to construct subgroups of the mapping class group isomorphic to the fundamental group of a closed surface. The mapping class group is weakly relatively hyperbolic [24], but is not strongly relatively hyperbolic [3], [7].

An open question by M. Gromov is whether every one-ended word hyperbolic group contains a subgroup isomorphic to the fundamental group of a closed surface. In [13], D. Cooper and D. Long produce surface subgroups in word hyperbolic groups arising as the fundamental groups of Dehn fillings of finite volume hyperbolic manifolds, starting with the construction of quasiconvex subgroups with particular structures in the finite volume hyperbolic manifold group. We aim to explore Gromov's question on particular classes of word hyperbolic groups which arise as algebraic Dehn fillings of relatively hyperbolic groups. The notion of algebraic Dehn filling has been studied by D. Groves and J. Manning, and independently by D. Osin [19], [26]. The main results of this paper are part of this program.

1.3. Other results on quasiconvex subgroups. Let $G$ be a hyperbolic group relative to a collection of subgroups $\mathscr{H}$ with finite generating set $X$. 
Proposition 1.3. Let $Q$ and $R$ be relatively quasiconvex subgroups of $G$. Then $Q \cap R$ is a relatively quasiconvex subgroup of $G$.

Remark 1.4. In the case of word hyperbolic groups, Proposition 1.3 was originally proved by H. Short. C. Hruska has independently proved this property without assuming that the ambient group is finitely generated [20]. In this generality, D. Osin stated the same result in [25].

Proposition 1.5. Let $Q$ be a $\sigma$-quasiconvex subgroup of $G$. The number of infinite maximal parabolic subgroups of $Q$ up to conjugacy in $Q$ is finite.

Remark 1.6. Proposition 1.5 is Theorem 9.1 of [20] and is proved using the dynamical characterization of quasiconvexity. Here we present a conceptually simpler proof using arguments on Cayley graphs.

1.4. Sample applications. Special attention has been given to relatively hyperbolic groups with peripheral structure consisting of abelian or virtually abelian subgroups. In this setting Theorems 1.1 and 1.2 can be used to construct quasiconvex subgroups with particular structures.

Let $G$ be hyperbolic relative to a collection of free abelian subgroups $\mathcal{H}$.

Given a subgroup $R$ of a group $Q$, the amalgamated free product of $k$ copies of $Q$ along $R$ is denoted by $\Delta_{m}(Q, R)$. When $m=2, \Delta_{2}(Q, R)$ is called the double of $Q$ along $K$. Doubling a group along a subgroup has been used in different contexts in group theory and geometric topology; for example to produce groups with interesting finiteness properties [28], or to produce surface subgroups in finite volume hyperbolic 3-manifold groups [12].

Corollary 1.7 (Doubling quasiconvex along parabolics). Let $Q$ be a relatively quasiconvex subgroup and let $P$ be a maximal parabolic subgroup of $G$. If

$$
\operatorname{rank}_{\mathbb{Z}}(Q \cap P)<\operatorname{rank}_{\mathbb{Z}}(P),
$$

then there exists a quasiconvex subgroup isomorphic to $\Delta_{k}(Q, Q \cap H)$ for any positive integer $k$.

A quasiconvex subgroup $R$ of $G$ is called fully quasiconvex if for any parabolic subgroup $P<G$, the subgroup $Q \cap P$ is finite or of finite index in $P$. Fully quasiconvex subgroups appear in the work of F. Dahmani [14] where it is shown that, under some hypothesis, the combination of relatively hyperbolic groups along fully quasiconvex subgroups is a relatively hyperbolic group.This class of subgroups also appeared in the the work by J. Manning and the author [22] to prove a consequence of the hypothetical absence of non-residually finite hyperbolic groups that was conjectured by I. Agol, D. Groves and J. Manning [1]. 
Corollary 1.8 (Fully quasiconvex amalgams). Let $Q$ be a relatively quasiconvex subgroup. Then there exists a fully quasiconvex subgroup $R$ which splits over $Q$.

1.5. Outline of the article. The article is organized as follows. Section 2 introduces background and notation. Section 3 states and proves a proposition about quasigeodesics which complements a result by C. Druţu and M. Sapir in [15]. Section 4 recalls the notion of relatively quasiconvex subgroup, and the proofs of Propositions 1.3 and 1.5 are explained. Section 5 consists of the proofs of the main results and the applications. The last section indicates directions of future research.

Acknowledgements. The results of this project are part of the author's PhD dissertation at the University of Oklahoma. He thanks his academic advisor Noel Brady for his guidance and encouragement, as well as Boris Apanasov, Ara Basmajian, Max Forester, Christopher Leininger, Pallavi Dani, Darryl McCullough, Krishnan Shankar, and Stephen Weldon for helpful comments during this work. The author is also grateful to the referee for several useful comments. This project was partially supported by NSF grant no. DMS-0505707 and the Department of Mathematics at the University of Oklahoma through a Foundation Fellowship.

\section{Relatively hyperbolic groups}

The aim of this section is to introduce notation and to define relatively hyperbolicity for finitely generated groups. The definition presented below is equivalent to the one given by D. Osin in [25]; the equivalence follows directly from [25], Theorems 3.23 and 7.10.

2.1. Preliminaries. We follow closely the notation and conventions of the paper [25]. Let $G$ be a group and $A \subset G$ a generating set closed under inverses. The Cayley graph of the group $G$ with respect to $A$, which is denoted by Cayley $(G, A)$, is the oriented graph with vertex set $G$ and edge set $G \times A$, where an edge $e=(g, a)$ goes from the vertex $g$ to the vertex $g a$ and has label $\operatorname{Label}(e)=a$. Let $p=$ $e_{1} e_{2} \ldots e_{k}$ be a combinatorial path in Cayley $(G, A)$. The initial and the terminal vertices of $p$ are denoted by $p_{-}$and $p_{+}$respectively, the label Label $(p)$ of $p$ is the word $\operatorname{Label}\left(e_{1}\right) \operatorname{Label}\left(e_{2}\right) \ldots \operatorname{Label}\left(e_{k}\right)$ in the alphabet $A$, and the length $l(p)$ of $p$ is the number of edges in $p$. The concatenation of the combinatorial paths $p$ and $q$ such that $p_{+}=q_{-}$is denoted by $p q$. The (word) length $|g|_{A}$ of an element $g \in G$ is the length of a shortest combinatorial path in $\operatorname{Cayley}(G, A)$ from 1 to $g$. This specifies a left invariant metric on the group $G$ defined by $\operatorname{dist}_{A}(f, g)=\left|f^{-1} g\right|_{A}$.

A geodesic metric space $(\Gamma, d)$ is a $\delta$-Gromov hyperbolic for some $\delta \geq 0$ if for any geodesic triangle $\Delta$, every side is contained in the open $\delta$-neighborhood of the other two sides. A rectifiable path $p$ in $(\Gamma, d)$ is a $(\lambda, c)$-quasi-geodesic for some 
$\lambda \geq 1$ and $c \geq 0$ if for any subpath $q$ of $p$,

$$
l(q) \leq \lambda d\left(q_{-}, q_{+}\right)+c .
$$

2.2. Definition of relative hyperbolicity. Let $G$ be a group, $\mathscr{H}=\left\{H_{i}\right\}_{i=1}^{m}$ be a collection of subgroups of $G$, and $X$ be a symmetric finite generating set for $G$. Denote by $\tilde{\mathscr{H}}$ the disjoint union

$$
\tilde{\mathscr{H}}=\bigsqcup_{i=1}^{m}\left(\tilde{H}_{i} \backslash\{1\}\right),
$$

where $\widetilde{H}_{i}$ is a copy of $H_{i}$.

Definition 2.1. The pair $\left(G,\left\{H_{i}\right\}_{i=1}^{m}\right)$ satisfies the weakly relative hyperbolicity condition if there is an integer $\delta \geq 0$ such that the Cayley graph Cayley $(G, X \cup \tilde{\tilde{H}})$ is a $\delta$-hyperbolic metric space.

Definition 2.2 (D. Osin [25]). Let $q$ be a combinatorial path in the Cayley graph Cayley $(G, X \cup \widetilde{\mathscr{H}})$. Subpaths of $q$ with at least one edge are called non-trivial. An $H_{i}$-component of $q$ is a maximal non-trivial subpath $s$ of $q$ with Label $(s)$ a word in the alphabet $\widetilde{H}_{i} \backslash\{1\}$. When we do not need to specify the index $i$, we will refer $H_{i}$-components as $\mathscr{H}$-components.

Two $\mathscr{H}$-components $s_{1}, s_{2}$ of $q$ are connected if the vertices of $s_{1}$ and $s_{2}$ belong to the same left coset of $H_{i}$ for some $i$. Equivalently, the $\mathscr{H}$-components $s_{1}$ and $s_{2}$ are connected if $\operatorname{Label}\left(s_{1}\right)$ and $\operatorname{Label}\left(s_{2}\right)$ are words in the alphabet $\widetilde{H}_{i}$ for some $i$, there exists a path $c$ which connects a vertex of $s_{1}$ and a vertex of $s_{2}$, and Label $(c)$ is a word in the alphabet $\widetilde{H}_{i}$. An $\mathscr{H}$-component $s$ of $q$ is isolated if it is not connected to a different $\mathscr{H}$-component of $q$.

The path $q$ is without backtracking if every $\mathscr{H}$-component of $q$ is isolated. A vertex $v$ of $q$ is called phase if it is not an inner vertex of an $\mathscr{H}$-component $s$ of $q$. Two paths $p$ and $q$ in $\operatorname{Cayley}(G, X \cup \widetilde{\mathscr{H}})$ are $k$-similar if

$$
\max \left\{d_{X}\left(p_{-}, q_{-}\right), d_{X}\left(p_{+}, q_{+}\right)\right\} \leq k .
$$

Remark 2.3. Every geodesic path in $\operatorname{Cayley}(G, X \cup \tilde{\mathscr{H}})$ is without backtracking and all its vertices are phase.

Definition 2.4. The pair $\left(G,\left\{H_{i}\right\}_{i=1}^{m}\right)$ satisfies the Bounded Coset Penetration (BCP) property if for any $\lambda \geq 1, c \geq 0, k \geq 0$, there exists an integer $\epsilon(\lambda, c, k)>0$ such that for any two $k$-similar $(\lambda, c)$-quasi-geodesics in $\operatorname{Cayley}(G, X \cup \tilde{\mathscr{H}})$ without backtracking $p$ and $q$ the following holds:

(i) The sets of phase vertices of $p$ and $q$ are contained in the closed $\epsilon(\lambda, c, k)$ neighborhoods (with respect to the metric dist $_{X}$ ) of each other. 
(ii) Suppose that $s$ is an $\mathscr{H}$-component of $p$ such that $\operatorname{dist}_{X}\left(s_{-}, s_{+}\right)>\epsilon(\lambda, c, k)$. Then there exists an $\mathscr{H}$-component $t$ of $q$ which is connected to $s$.

(iii) Suppose that $s$ and $t$ are connected $\mathscr{H}$-components of $p$ and $q$, respectively. Then $\max \left\{\operatorname{dist}_{X}\left(s_{-}, t_{-}\right), \operatorname{dist}_{X}\left(s_{+}, t_{+}\right)\right\} \leq \epsilon(\lambda, c, k)$.

Remark 2.5. Our definition of the BCP property corresponds to the conclusion of Theorem 3.23 in [25].

Definition 2.6. The pair $\left(G,\left\{H_{i}\right\}_{i=1}^{m}\right)$ satisfies the relative hyperbolicity condition if the group $G$ is weakly hyperbolic relative to $\left\{H_{i}\right\}_{i=1}^{m}$ and the pair $\left(G,\left\{H_{i}\right\}_{i=1}^{m}\right)$ satisfies the Bounded Coset Penetration property. If $\left(G,\left\{H_{i}\right\}_{i=1}^{m}\right)$ satisfies the relative hyperbolicity condition then we said that group $G$ is hyperbolic relative to $\left\{H_{i}\right\}_{i=1}^{m}$; if there is no ambiguity, we just said that the group $G$ is relatively hyperbolic.

Remark 2.7. The stated definition of relative hyperbolicity is equivalent to Definition 2.35 in [25] for finitely generated groups. This follows directly from [25], Theorems 3.23 and 7.10 .

For the rest of this section, let $G$ be a group hyperbolic relative to a collection of subgroups $\mathscr{H}$ and let $X$ be a symmetric finite generating set of $G$.

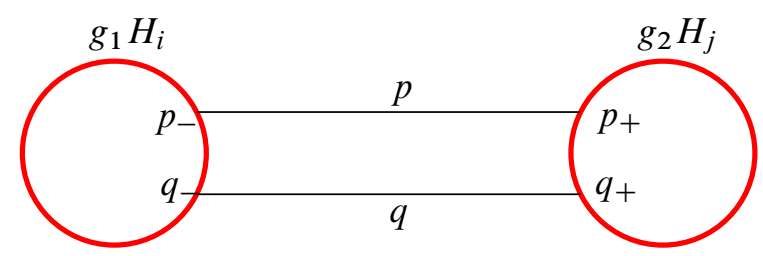

Figure 1. Two geodesics connecting different left cosets $g_{1} H_{i}$ and $g_{2} H_{j}$ are $\epsilon(1,4,0)$-similar, and their lengths differ by at most two (Corollary 2.8).

The following corollary is a direct consequence of Definition 2.6.

Corollary 2.8. Let $g_{1} H_{i}$ and $g_{2} H_{j}$ be different left cosets. For any pair of geodesics $p$ and $q$ in Cayley $(G, X \cup \tilde{\mathscr{H}})$ such that $p_{-}, q_{-} \in g_{1} H_{i}, p_{+}, q_{+} \in g_{2} H_{j}$, and neither $p$ nor $q$ have more than one vertex in $g_{1} H_{i}$ or $g_{2} H_{j}$, the following holds.

(1) $l(q) \leq l(p)+2$, and

(2) $q$ and $p$ are $\epsilon(1,4,0)$-similar.

Proof. Consider the path $r=c_{1} p c_{2}$ in $\operatorname{Cayley}(G, X \cup \tilde{\mathscr{H}})$ where $c_{1}$ is an edge connecting $q_{-}$and $p_{-}$, and $c_{2}$ is an edge connecting $p_{+}$and $q_{+}$. Notice that $r$ is a $(1,4)$-quasi-geodesic in $\operatorname{Cayley}(G, X \cup \tilde{\mathscr{H}})$ and that $q$ and $r$ are 0 -similar. The BCP property implies

$$
\max \left\{\operatorname{dist}_{X}\left(\left(c_{1}\right)_{-},\left(c_{1}\right)_{+}\right), \operatorname{dist}_{X}\left(\left(c_{2}\right)_{-},\left(c_{2}\right)_{+}\right)\right\} \leq \epsilon(1,4,0) .
$$




\section{Quasi-geodesics}

Let $G$ be a group generated by a finite set $X$, let $\left\{H_{i}\right\}_{i=1}^{m}$ be a collection of subgroups of $G$, and suppose that $G$ is hyperbolic relative to $\left\{H_{i}\right\}_{i=1}^{m}$. Any geodesic $p$ in Cayley $(G, X \cup \tilde{\mathscr{H}})$ can be decomposed as

$$
p=r_{1} s_{1} \ldots r_{k} s_{k},
$$

where each $r_{i}$ is a geodesic and each $s_{i}$ is an isolated $\mathscr{H}$-component.

In this section, we investigate paths with the above type of decomposition and estimate quasi-geodesic constants.

The main result of the section is the following.

Proposition 3.1. There are constants $\eta$ and $\lambda_{0}$ with the following properties. If $p$ is a path in Cayley $(G, X \cup \widetilde{\mathscr{H}})$ such that

(1) $p=r_{1} s_{1} \ldots r_{k} s_{k}$, where each $r_{i}$ and $s_{i}$ are geodesic paths in Cayley $(G, X \cup \tilde{\mathscr{H}})$,

(2) the subpath $s_{i}$ of $p$ is an $\mathscr{H}$-component of $p$ for each $i$,

(3) $\operatorname{dist}_{X}\left(\left(s_{i}\right)_{-},\left(s_{i}\right)_{+}\right) \geq \eta$ for each $i$, and

(4) the $\mathscr{H}$-components $s_{i}$ and $s_{i+1}$ of $p$ are not connected for each $i$.

Then $p$ is a $\left(\lambda_{0}, 0\right)$-quasi-geodesic without backtracking and with different endpoints.

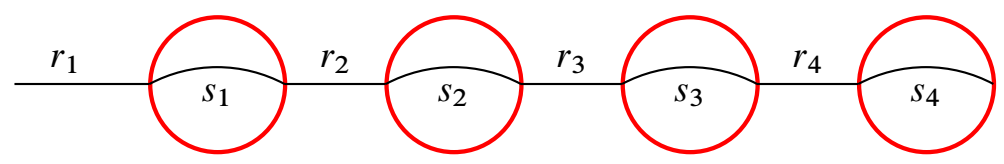

Figure 2. Polygonal path $p$ in Proposition 3.1.

Remark 3.2. The strength of this result is that the constant $\lambda_{0}$ is independent of the number of segments of the path. This complements a similar result by C. Druţu and M. Sapir [15], Lemma 8.12. Assuming that the lengths of the segments $r_{i}$ are larger than a fixed constant $l$, their result estimates quasi-geodesic constants depending on $l$ and the number of segments $2 k$.

The rest of the section consists of two parts. First, a result by D. Osin about polygons in Cayley $(G, X \cup \tilde{\mathscr{H}})$ is recalled and a corollary is stated. The second part consists of a series of lemmas and the proof of Proposition 3.1.

3.1. Osin's result about polygons. The following proposition is a stronger version of the Bounded Coset Penetration property. It is a central part of D. Osin's work in [26]. 
Proposition 3.3 (D. Osin [26]). There exists a constant $D>0$ satisfying the following condition. If $\mathcal{P}=p_{1} p_{2} \ldots p_{n}$ is an $n$-gon in $\operatorname{Cayley}(G, X \cup \widetilde{\mathscr{H}})$ and $S \subset\left\{p_{1}, \ldots, p_{n}\right\}$ such that

(1) each side $p_{i} \in S$ is an isolated $\mathscr{H}$-component of $\mathcal{P}$, and

(2) each side $p_{i} \notin S$ is a geodesic path.

Then

$$
\sum_{p \in S} \operatorname{dist}_{X}\left(p_{-}, p_{+}\right) \leq D n
$$

The next corollary is used in the proof of the main result of the section.

Corollary 3.4. There exists a constant $\tau \geq 0$ with the following property. Let $\Delta=$ $p q r$ be a triangle whose sides $p, q, r$ are geodesics in Cayley $(G, X \cup \widetilde{\mathscr{H}})$. If $s$ is an $\mathscr{H}$-component of $p$, then either

(1) $s$ is an isolated $\mathscr{H}$-component of the cycle $\Delta$ and $\operatorname{dist}_{X}\left(s_{-}, s_{+}\right) \leq \tau$;

(2) $s$ is connected to only one $\mathscr{H}$-component $t$ of the concatenated path qr and

$$
\max \left\{\operatorname{dist}_{X}\left(s_{-}, t_{+}\right), \operatorname{dist}_{X}\left(s_{+}, t_{-}\right)\right\} \leq \tau ;
$$

(3) $s$ is connected to $\mathscr{H}$-components $t$ and $u$ of $q$ and $r$ respectively, and

$$
\max \left\{\operatorname{dist}_{X}\left(s_{+}, t_{-}\right), \operatorname{dist}_{X}\left(t_{+}, u_{-}\right), \operatorname{dist}_{X}\left(u_{+}, s_{-}\right)\right\} \leq \tau .
$$
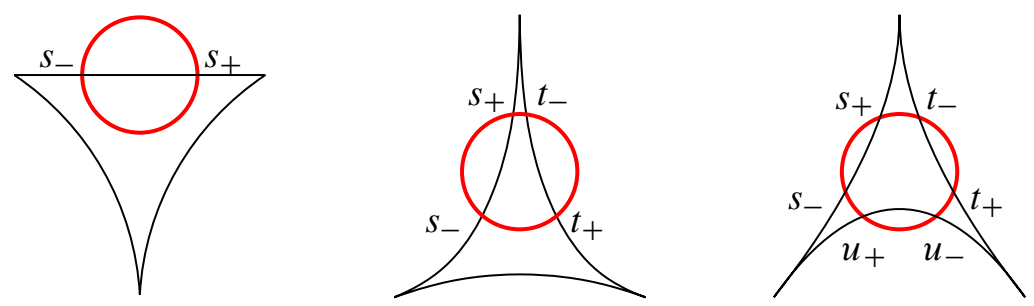

Figure 3. The three cases of Corollary 3.4.

Proof. In the first case, consider $\Delta$ as a 5-gon with $s$ as one of its sides. Then Proposition 3.3 implies that $\operatorname{dist}_{X}\left(s_{-}, s_{+}\right) \leq 5 D$.

In the second case, we consider the case $t$ is an $\mathscr{H}$-component of $q$. Decompose the paths $p$ and $q$ as $p=p_{1} s p_{2}$ and $q=q_{1} t q_{2}$. Since $s$ and $t$ are connected $\mathscr{H}$ components, there are edges $c_{1}$ connecting $t_{-}$and $s_{+}$, and $c_{2}$ connecting $s_{-}$and $t_{+}$. Considering the 3-gon $p_{2} q_{1} c_{1}$ and the 4-gon $p_{1} c_{2} q_{2} r$, Proposition 3.3 implies that

$$
\max \left\{\operatorname{dist}_{X}\left(s_{-}, t_{+}\right), \operatorname{dist}_{X}\left(s_{+}, t_{-}\right)\right\} \leq 4 D .
$$


For the third case, an analogous argument shows that

$$
\max \left\{\operatorname{dist}_{X}\left(s_{+}, t_{-}\right), \operatorname{dist}_{X}\left(t_{+}, u_{-}\right), \operatorname{dist}_{X}\left(u_{+}, s_{-}\right)\right\} \leq 3 D
$$

To finish the proof define $\tau=5 D$.

Remark 3.5. An equivalent result to Corollary 3.4 appears as [15], Proposition 8.16.

3.2. Proof of Proposition 3.1. We will see that $\lambda_{0}=3$ works and define a lower bound for $\eta$ during the course of the proof. The argument consists of three lemmas.

Lemma 3.6. Let $p$ be a path satisfying the hypothesis of Proposition 3.1, and let $q$ be a geodesic in Cayley $(G, X \cup \widetilde{\mathscr{H}})$ connecting the endpoints of $p$. The $q$ contains, for each $i$, an $\mathscr{H}$-component $t_{i}$ satisfying

(1) $s_{i}$ and $t_{i}$ are connected $\mathscr{H}$-components, and

(2) $s_{i}$ and $t_{i}$ are $\epsilon(1,4,0)$-similar.

In particular, the endpoints of p are different (see Figure 4).

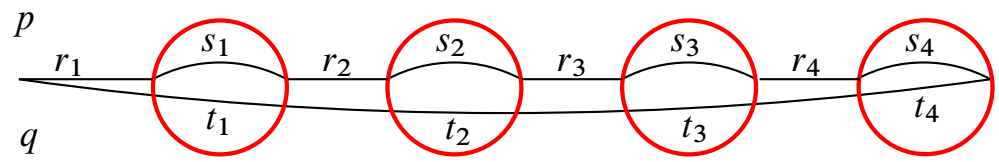

Figure 4. The path $p$ and a geodesic $q$ connecting the endpoints of $p$.

Proof. We argue by induction on $k$. Suppose that $k=1$. Since $l\left(s_{1}\right)=1$ it follows that $r_{1} s_{1}$ is a $(1,2)$-quasi-geodesic in $\operatorname{Cayley}(G, X \cup \tilde{\mathscr{H}})$. By our choice of $\eta$,

$$
\operatorname{dist}_{X}\left(\left(s_{1}\right)_{-},\left(s_{1}\right)_{+}\right) \geq \eta>\epsilon(1,2,0) .
$$

The BCP property implies that any geodesic in $\operatorname{Cayley}(G, X \cup \tilde{\mathscr{H}})$ connecting the endpoints of $r_{1} s_{1}$ has an $\mathscr{H}$-component which is connected to $s_{1}$ and is $\epsilon(1,2,0)$ similar to $s_{1}$.

Suppose that $k>1$. Consider the subpaths of $p$ :

$$
p_{1}=r_{1} s_{1} \ldots r_{k-1} s_{k-1} \text { and } p_{2}=r_{k} s_{k}
$$

Let $q, q_{1}$, and $q_{2}$ be geodesics in $\operatorname{Cayley}(G, X \cup \tilde{\mathscr{H}})$ connecting the endpoints of $p$, $p_{1}$ and $p_{2}$, respectively.

Claim 1. $q_{1}$ has no $\mathscr{H}$-component connected to the $\mathscr{H}$-component $s_{k}$. 
By induction hypothesis $q_{1}$ has an $\mathscr{H}$-component $u$ which is connected and $\epsilon(1,4,0)$-similar to $s_{k-1}$. By the triangle inequality and our choice of $\eta$,

$$
\begin{aligned}
\operatorname{dist}_{X}\left(u_{-}, u_{+}\right) & \geq \operatorname{dist}_{X}\left(\left(s_{k-1}\right)_{-},\left(s_{k-1}\right)_{+}\right)-2 \epsilon(1,4,0) \\
& \geq \eta-2 \epsilon(1,4,0) \\
& >\epsilon(1,4,0) .
\end{aligned}
$$

Suppose that $q_{1}$ has an $\mathscr{H}$-component $v$ connected to $s_{k}$ (see Figure 5). Consider the subpath $\left[v_{+}, u_{-}\right]_{q_{1}}$ of $q_{1}$ from $v_{+}$to $u_{-}$. Corollary 2.8 implies that $\left[v_{+}, u_{-}\right]_{q_{1}}$ and $r_{k}^{-1}$ are $\epsilon(1,4,0)$-similar paths. In particular $\operatorname{dist}_{X}\left(u_{-}, u_{+}\right) \leq \epsilon(1,4,0)$ which contradicts (1).

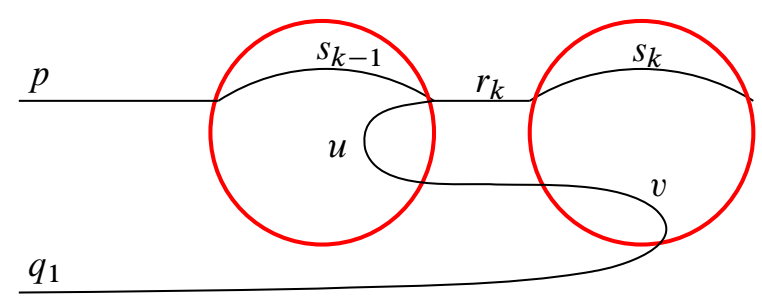

Figure 5. Claim 1 in Lemma 3.6. If $q_{1}$ has an $\mathscr{H}$-component $v$ connected to $s_{k}$, then dist $_{X}\left(u_{-}, u_{+}\right) \leq \epsilon(1,4,0)$, which is a contradiction.

Claim 2. $q$ has an $\mathscr{H}$-component $t_{k}$ connected to $s_{k}$.

Consider the triangle $\Delta$ whose sides are $q_{1}, q_{2}$, and $q$. By induction hypothesis, $q_{2}$ has an $\mathscr{H}$-component $t$ which is connected and $\epsilon(1,4,0)$-similar to $s_{k}$. By the triangle inequality and our choice of $\eta$,

$$
\operatorname{dist}_{X}\left(t_{-}, t_{+}\right) \geq \eta-2 \epsilon(1,4,0)>\tau,
$$

where $\tau$ is the constant of Corollary 3.4. Since $q_{1}$ has no $\mathscr{H}$-component connected to $s_{k}$, Corollary 3.4 implies that $q$ has an $\mathscr{H}$-component $t_{k}$.

Claim 3. $q$ has an $\mathscr{H}$-component $t_{k-1}$ connected to $s_{k-1}$.

Since $p_{2}$ and $q_{2}$ are 0 -similar, if $q_{2}$ has an $\mathscr{H}$-component $t$ connected to $s_{k-1}$, then the BCP property shows that $\operatorname{dist}_{X}\left(t_{-}, t_{+}\right) \leq \epsilon(1,2,0)$. By the induction hypothesis, the $\mathscr{H}$-component $u$ of $q_{1}$ connected to $s_{k-1}$ satisfies dist ${ }_{X}\left(u_{-}, u_{+}\right) \geq \eta-2 \epsilon(1,4,0)$. Consider the triangle $\Delta$ whose sides are $q_{1}, q_{2}$, and $q$. Corollary 3.4 implies that $q$ has an $\mathscr{H}$-component $t_{k-1}$ connected to $s_{k-1}$ such that

$$
\operatorname{dist}_{X}\left(\left(t_{k-1}\right)_{-},\left(t_{k-1}\right)_{+}\right) \geq \eta-2 \epsilon(1,4,0)-\epsilon(1,2,0)-3 \tau>0 .
$$

The last inequality follows by our choice of $\eta$.

Claim 4. $q$ contains, for each $1 \leq i<k-1$, an $\mathscr{H}$-component $t_{i}$ which is connected to $s_{i}$. 
Let $q^{\prime}$ be the subpath of $q$ from $q_{-}$to $\left(t_{k-1}\right)_{+}$. Notice that $q^{\prime}$ and $q_{1}$ are $\epsilon(1,4,0)$ similar (apply Corollary 2.8 to $r_{k}$ and the subpath of $q$ from $\left(t_{k-1}\right)_{+}$to $\left.\left(t_{k}\right)_{-}\right)$. By induction hypothesis $q_{1}$ has an $\mathscr{H}$-component $u_{i}$ connected to $s_{i}$ such that

$$
\operatorname{dist}_{X}\left(\left(u_{i}\right)_{-},\left(u_{i}\right)_{+}\right) \geq \eta-2 \epsilon(1,2,0)>\epsilon(1,0, \epsilon(1,4,0)),
$$

where the last inequality follows by our choice of $\eta$. Applying the BCP property to $q^{\prime}$ and $q_{1}$, one sees that $q^{\prime}$ has an $\mathscr{H}$-component $t_{i}$ connected to $s_{i}$ such that

$$
\operatorname{dist}_{X}\left(\left(t_{i}\right)_{-},\left(t_{i}\right)_{+}\right) \geq \eta-2 \epsilon(1,2,0)-2 \epsilon(1,0, \epsilon(1,4,0))>0,
$$

where the last inequality follows by our choice of $\eta$. The claim follows.

Claim 5. The $\mathscr{H}$-components $s_{i}$ and $t_{i}$ of $p$ and $q$, respectively, are $\epsilon(1,4,0)$ similar.

Let $w_{i}$ be the subpath of $q$ between $t_{i}$ and $t_{i+1}$. Corollary 2.8 implies that $r_{i}$ and $w_{i}$ are $\epsilon(1,4,0)$-similar for each $i$. This completes the proof of the lemma.

Lemma 3.7. Let $p$ be a path satisfying the hypothesis of Proposition 3.1. Then $p$ is $a(3,0)$-quasi-geodesic.

Proof. Let $p^{\prime}$ be a subpath of $p$ and let $q^{\prime}$ a geodesic in Cayley $(G, X \cup \tilde{\mathscr{H}})$ connecting the endpoints of $p^{\prime}$. The Lemma above implies that $q^{\prime}$ has an $\mathscr{H}$-component $t_{i}$ connected to the $\mathscr{H}$-component $s_{i}$ of $p^{\prime}$. Therefore we have decompositions of the two paths of the form:

$$
\begin{aligned}
& p^{\prime}=r_{l}^{\prime} s_{l} r_{l+1} \ldots r_{l+\jmath} s_{l+\jmath} r_{l+\jmath+1}^{\prime}, \\
& q^{\prime}=u_{l} t_{l} u_{l+1} \ldots u_{l+\jmath} t_{l+\jmath} u_{l+J+1} .
\end{aligned}
$$

Corollary 2.8 implies that $l\left(r_{i}\right) \leq l\left(u_{i}\right)+2$ for $i=l+1 \ldots l+J, l\left(r_{l}^{\prime}\right) \leq l\left(u_{l}\right)+2$, and $l\left(r_{l+\jmath+1}^{\prime}\right) \leq l\left(u_{l+\jmath+1}\right)$. It follows that

$$
l\left(p^{\prime}\right) \leq l\left(u_{l}\right)+2+\sum_{i=l+1}^{J+J}\left(l\left(s_{i}\right)+l\left(u_{i+1}\right)+2\right)+l\left(u_{l+\jmath+1}\right)+2 \leq 3 l\left(q^{\prime}\right)
$$

It follows that $p$ is a $(3,0)$-quasi-geodesic in $\operatorname{Cayley}(G, X \cup \tilde{\mathscr{H}})$ since $p^{\prime}$ was arbitrary.

Lemma 3.8. Let $p$ be a path satisfying the hypothesis of Proposition 3.1. Then $p$ is without backtracking.

Proof. Suppose that $p$ backtracks. Let $u$ and $v$ be different connected $\mathscr{H}$-components of $p$ such that the subpath $r$ in between is without backtracking. Observe that $r$ 
contains one of the $\mathscr{H}$-components $s_{i}$ of $p$. Since $r$ is a $(3,0)$-quasi-geodesic, the $\mathrm{BCP}$ property implies that

$$
\operatorname{dist}_{X}\left(\left(s_{i}\right)_{-},\left(s_{i}\right)_{+}\right) \leq \epsilon(3,0,0) .
$$

But this is a contradiction since

$$
\operatorname{dist}_{X}\left(\left(s_{i}\right)_{-},\left(s_{i}\right)_{+}\right) \geq \eta>\epsilon(3,0,0),
$$

by our choice of $\eta$.

A lower bound for $\eta$ is given by (1), (2), (3), (4), (5), and (6).

\section{Quasiconvex subgroups}

This section consists of three parts. First (relatively) quasiconvex subgroups are defined following D. Osin's work in [25]. The second part consists of the proof of Proposition 1.3, which states that the collection of quasiconvex subgroups of a relatively hyperbolic group is closed under finite intersections. The third part is devoted to the proof of Proposition 1.5 on maximal parabolic subgroups of quasiconvex subgroups.

In this section, $G$ is a group generated by a finite set $X,\left\{H_{i}\right\}_{i=1}^{m}$ a collection of subgroups of $G$, and $G$ is hyperbolic relative to $\left\{H_{i}\right\}_{i=1}^{m}$.

4.1. Definition of relatively quasiconvex subgroups. Relatively quasiconvex subgroups of relatively hyperbolic groups were introduced by D. Osin in [25] as a generalization of quasiconvex subgroups of word hyperbolic groups. F. Dahmani in [14] introduced a dynamical definition of quasiconvex subgroups in relatively hyperbolic groups. C. Hruska showed that both notions are equivalent [20].

Definition 4.1 (D. Osin [25]). A subgroup $Q$ of $G$ is called quasiconvex relative to $\left\{H_{i}\right\}_{i=1}^{m}$ (or simply quasiconvex when the collection $\left\{H_{i}\right\}_{i=1}^{m}$ is fixed) if there exists a constant $\sigma \geq 0$ such that the following condition holds:

Let $f, g$ be two elements of $Q$, and let $p$ be an arbitrary geodesic path from $f$ to $g$ in Cayley $(G, X \cup \tilde{\mathscr{H}})$. Then for any vertex $v \in p$, there exists a vertex $w \in Q$ such that $\operatorname{dist}_{X}(u, w) \leq \sigma$.

4.2. Proof of Proposition 1.3. The following lemma is used several times in the paper, in particular in the proof of Proposition 1.3. In the context of countable groups, we say that a left invariant metric is proper if balls of finite radius contain a finite number of elements. 
Lemma 4.2. Let $A$ be a countable group with a proper left invariant metric $d$. Then for any subgroups $B$ and $C$ of $A$, and any constant $K \geq 0$, there exists $M=$ $M(B, C, K) \geq 0$ so that

$$
B \cap N_{K}(C) \subset N_{M}(B \cap C),
$$

where $N_{K}(C)$ and $N_{M}(B \cap C)$ denote the closed $K$-neighborhood and the closed $M$-neighborhood of $C$ and $B \cap C$ in $(A, d)$ respectively.

Proof. Suppose that the statement is false for the constant $K$. Then there are sequences $\left\{q_{n}\right\}_{n=1}^{\infty}$ and $\left\{h_{n}\right\}_{n=1}^{\infty}$ such that $q_{n} \in B, q_{n} h_{n} \in C, d\left(1, h_{n}\right) \leq K$, and

$$
d\left(q_{n}, B \cap C\right) \geq n .
$$

Since balls are finite in the metric space $(A, d)$, without lost of generality assume $\left\{h_{n}\right\}_{n=1}^{\infty}$ is a constant sequence $\{h\}_{n=1}^{\infty}$. For any $m$ and $n$, observe that $q_{n} q_{m}^{-1}=$ $\left(q_{n} h\right)\left(q_{m} h\right)^{-1} \in B \cap C$, and hence $q_{m} h$ and $q_{n} h$ are in the same right coset of $B \cap C$, say $(B \cap C) f$. It follows that

$$
d\left(q_{n}, B \cap C\right) \leq d\left(q_{n}, q_{n} h\right)+d\left(q_{n} h, B \cap C\right) \leq K+d(1, f)
$$

for any $n$, a contradiction.

Remark 4.3. A more general result than Lemma 4.2 appears in [20], Proposition 9.4.

Proof of Proposition 1.3. Let $\sigma>0$ so that $Q$ and $R$ are $\sigma$-quasiconvex relative to $\left\{H_{i}\right\}_{i=1}^{m}$. Since the generating set $X$ is finite, the metric $\operatorname{dist}_{X}$ on $G$ is proper. Let $M=M(Q, R, 2 \sigma)$ be the constant given by Lemma 4.2 satisfying

$$
Q \cap N_{2 \sigma}(R) \subset N_{M}(Q \cap R),
$$

where the neighborhoods are taken in the metric space $\left(G\right.$, dist $\left._{X}\right)$.

We claim that $Q \cap R$ is a $(\sigma+M)$-quasiconvex relative to $\left\{H_{i}\right\}_{i=1}^{m}$. Let $g \in Q \cap R$, let $p$ be a geodesic from 1 to $g$ in $\operatorname{Cayley}(G, X \cup \tilde{\mathscr{H}})$, and let $u$ be a vertex of $p$. Since $Q$ and $R$ are $\sigma$-quasiconvex, there exists $s \in Q$ and $t \in R$ so that

$$
\max \left\{\operatorname{dist}_{X}(s, u), \operatorname{dist}_{X}(t, u)\right\} \leq \sigma .
$$

It follows that $s \in Q \cap N_{2 \sigma}(R)$, and hence there is $v \in Q \cap R$ so that dist $X(s, v) \leq M$. Therefore $v \in Q \cap R$ and $\operatorname{dist}_{X}(u, v) \leq \sigma+M$. 


\subsection{Proof of Proposition 1.5}

Proposition 1.5. Let $Q$ be a $\sigma$-quasiconvex subgroup of $G$. Then any infinite maximal parabolic subgroup of $Q$ is conjugate by an element of $Q$ to a subgroup in the set

$$
\left\{Q \cap H^{z} \mid H \in \mathscr{H} \text { and } z \in G \text { with }|z|_{X} \leq \sigma\right\} .
$$

In particular, the number of infinite maximal parabolic subgroups up to conjugacy in $Q$ is finite.

Proof. Let $g \in G$ and $H \in \mathscr{H}$. Suppose that $Q \cap H^{g}$ is an infinite subgroup. Since the generating set $X$ of $G$ is finite, there is an element $h \in H$ such that $|h|_{X}>\epsilon\left(1,0,|g|_{X}\right)$ and $h^{g} \in Q \cap H^{g}$. Let $p$ be a geodesic from 1 to $h^{g}$. Then the BCP property 2.4 implies that $p$ has an $\mathscr{H}$-component $s$ contained in the left $\operatorname{coset} g H$. Since $Q$ is $\sigma$-quasiconvex, there is an element $y \in Q$ such that $\operatorname{dist}_{X}\left(y, s_{-}\right) \leq \sigma$. The group element $z=y^{-1} S_{-}$satisfies $|z|_{X} \leq \sigma$ and

$$
\left(Q \cap H^{z}\right)^{y}=Q \cap H^{s_{-}}=Q \cap H^{g} .
$$

\section{Proofs of the combination theorems and applications}

The proofs of Theorems 1.1 and 1.2 adapt some of Gromov's ideas in [18], Section 5.3.C, on combination theorems for quasiconvex subgroups in word hyperbolic groups. We sketch the general argument. Suppose that $Q * Q \cap R R$ is an amalgamated product of quasiconvex subgroups of $G$ satisfying the conditions of one of main theorems. Given a non-trivial element $f$ of $Q * Q \cap R R$, we use its normal form to produce a path $o$ in the relative Cayley graph of $G$ from 1 to the image of $f$. Then the path $o$ is shortened by replacing each $\mathscr{H}$-component with more than one edge by a single edge; the new path is denoted by $p$. (See Figure 7 below.) Proposition 3.1 implies that $p$ is a $\left(\lambda_{0}, 0\right)$-quasi-geodesic with different endpoints, proving that the map from $Q * Q \cap S S$ into $G$ is injective. Since $\lambda_{0}$ is independent of the element $f$, the image of $Q * Q \cap S S$ in $G$ will be a quasiconvex subgroup.

The section consists of four parts. In the first part a proof of Theorem 1.1 is explained in detail. Then the proof of Theorem 1.2 is discussed. The last two parts correspond to the proof of Corollaries 1.7 and 1.8.

5.1. Proof of Theorem 1.1. Conjugate the subgroup $Q$ if necessary and assume that $P=H$ for some $H \in \mathscr{H}$. A lower bound for the constant $C$ is defined during the course of the proof, in particular, the constant $C$ is chosen large enough to satisfy (8) below. The proof consists of four lemmas. Let $\sigma$ be the quasiconvexity constant for $Q$. 
Lemma 5.1. The natural homomorphism $Q * Q \cap R R \rightarrow G$ is injective.

Proof. Let $f$ be a non-trivial element of $Q * Q \cap R R$. If $f$ is conjugate to an element of $Q$ or an element of $R$, then it is clear that its image is not trivial. Otherwise,

$$
f=g_{1} h_{1} \ldots g_{k} h_{k},
$$

where $g_{i} \in Q \backslash Q \cap R$ for $1<i \leq k, h_{i} \in R \backslash Q \cap R$ for $1 \leq i<k$, either $g_{1}=1$ or $g_{1} \in Q \backslash Q \cap R$, and either $h_{k}=1$ or $h_{k} \in R \backslash Q \cap R$. Since $f$ is not conjugate to an element of $Q$ or $R$, after conjugating if necessary, assume that $g_{1} \neq 1$ and $h_{k} \neq 1$. Consider the path $o$ in $\operatorname{Cayley}(G, X \cup \tilde{\mathscr{H}})$ given by

$$
o=u_{1} v_{1} \ldots u_{k} v_{k},
$$

where each $u_{i}$ and $v_{i}$ are geodesic paths in $\operatorname{Cayley}(G, X \cup \tilde{\mathscr{H}})$, Label $\left(u_{i}\right)$ represents $g_{i}$, and Label $\left(v_{i}\right)$ represents $h_{i}$.

Claim 1 . Let $t_{i}$ be the $\mathscr{H}$-component of the path $o$ containing the subpath $v_{i}$. Then the $\mathscr{H}$-components $t_{i}$ and $t_{i+1}$ are not connected.

If $t_{i}$ and $t_{i+1}$ are connected, then Label $\left(u_{i}\right)$ represents an element of $R$. But this contradicts the assumptions on the normal form (7) of the element $f$.

Claim 2. For each $i$, dist $X_{X}\left(\left(t_{i}\right)_{-},\left(t_{i}\right)_{+}\right)>\eta$, where $\eta$ is the constant from Proposition 3.1.

Fix $i$ and let $x_{1}=\left(u_{i}\right)_{-}, x_{2}=\left(t_{i}\right)_{-}, x_{3}=\left(u_{i}\right)_{+}, x_{4}=\left(u_{i+1}\right)_{-}, x_{5}=\left(t_{i}\right)+$, and $x_{6}=\left(u_{i+1}\right)_{+}$. (See Figure 6.)

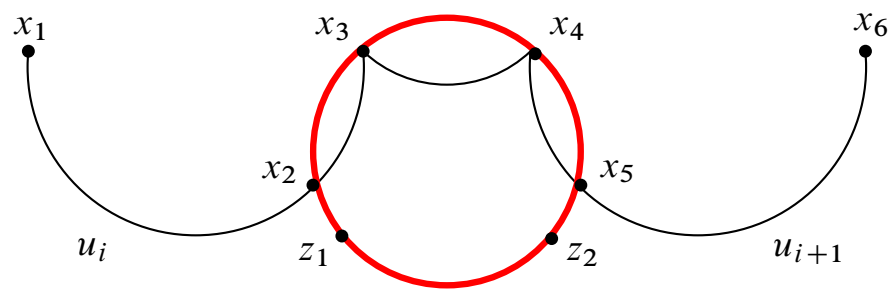

Figure 6. The endpoints of $v_{i}$ are $x_{3}$ and $x_{4}$. The $\mathscr{H}$-component $t_{i}$ of $o$ containing $v_{i}$ consists of the path $\left[x_{2}, x_{3}\right] v_{i}\left[x_{4}, x_{5}\right]$.

First we show that there are elements $z_{1}$ and $z_{2}$ in the left coset $x_{3}(H \cap Q)=$ $x_{4}(H \cap Q)$ such that

$$
\max \left\{\operatorname{dist}_{X}\left(x_{2}, z_{1}\right), \operatorname{dist}_{X}\left(x_{5}, z_{2}\right)\right\} \leq M(H, Q, \sigma),
$$

where $M(H, Q, \sigma)$ is the constant provided by Lemma 4.2 for the subgroups $H$ and $Q$, the constant $\sigma$, and the proper metric $\operatorname{dist}_{X}$. Since $Q$ is $\sigma$-quasiconvex and $\operatorname{Label}\left(u_{i}\right)$ 
represents an element of $Q$, we have that $x_{3}^{-1} x_{2} \in H$ and $d_{X}\left(x_{3}^{-1} x_{2}, Q\right) \leq \sigma$; then Lemma 4.2 implies that

$$
d_{X}\left(x_{3}^{-1} x_{2}, H \cap Q\right) \leq M(H, Q, \sigma) ;
$$

hence there is an element $z_{1} \in x_{3}(H \cap Q)$ such that $d_{X}\left(x_{2}, z_{1}\right) \leq M(H, Q, \sigma)$. A similar argument guarantees the existence of an element $z_{2}$ with the desire properties.

Since $x_{3}^{-1} x_{4} \in R \backslash Q, x_{3}^{-1} z_{1} \in(H \cap Q) \subset R$, and $x_{4}^{-1} z_{2} \in(H \cap Q) \subset R$, we have that

$$
z_{1}^{-1} z_{2}=\left(z_{1}^{-1} x_{3}\right)\left(x_{3}^{-1} x_{4}\right)\left(x_{4}^{-1} z_{2}\right) \in R \backslash Q,
$$

and hence $\operatorname{dist}_{X}\left(z_{1}, z_{2}\right) \geq C$ by hypothesis (2) on the length of the elements of $R \backslash Q$. Finally, by our choice of the constant $C$,

$$
\begin{aligned}
\operatorname{dist}_{X}\left(x_{2}, x_{5}\right) & \geq \operatorname{dist}_{X}\left(z_{1}, z_{2}\right)-\operatorname{dist}_{X}\left(x_{2}, z_{1}\right)-\operatorname{dist}_{X}\left(x_{5}, z_{2}\right) \\
& \geq C-2 M(H, Q, \sigma) \\
& \geq \eta
\end{aligned}
$$

which proves the claim.

Claim 3. Let $p$ be the path obtained by replacing each $\mathscr{H}$-component $t_{i}$ of $o$ for a single edge $s_{i}$. (See Figure 7.) Then $p$ is $\left(\lambda_{0}, 0\right)$-quasi-geodesic with different

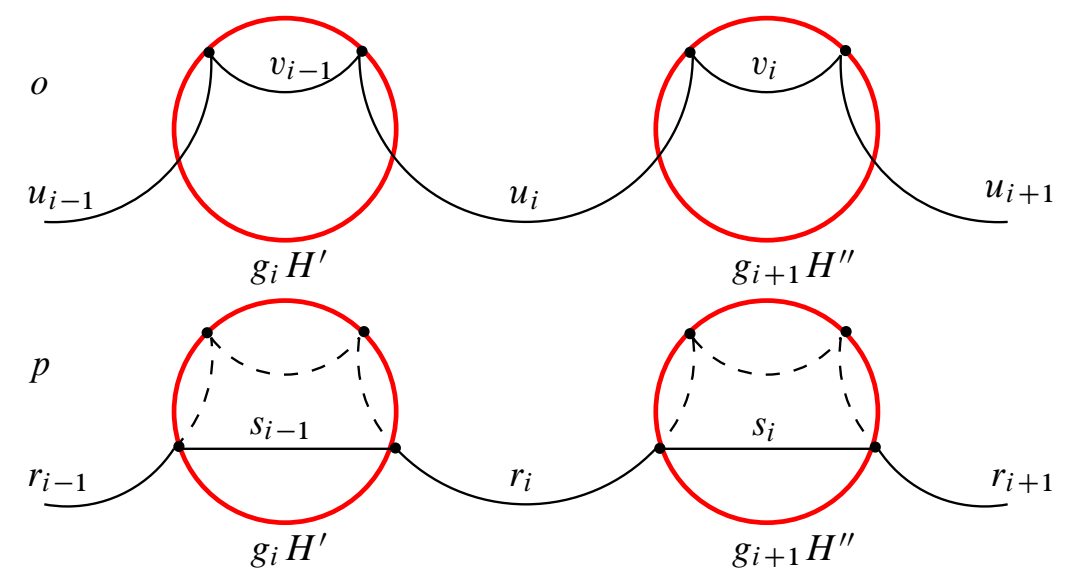

Figure 7. The path $o$ and the resulting quasi-geodesic $p$.

endpoints. In particular the image of $f$ by the map $Q * Q \cap R R \rightarrow G$ is not trivial. The path $p$ has a decomposition of the form

$$
p=r_{1} s_{1} \ldots r_{k} s_{k}
$$


where $r_{1}$ or $s_{k}$ may be trivial. The definition of the path $p$ (and the path $o$ ) shows that $r_{i}$ and $s_{i}$ are geodesic segments in $\operatorname{Cayley}(G, X \cup \tilde{\mathscr{H}})$; Claim 1 shows that the $\mathscr{H}$-components $s_{i}$ and $s_{i+1}$ of $p$ are not connected; and Claim 2 implies that

$$
\operatorname{dist}_{X}\left(\left(s_{i}\right)_{-},\left(s_{i}\right)_{+}\right) \geq \eta .
$$

Proposition 3.1 implies that $p$ is a $\left(\lambda_{0}, 0\right)$-quasi-geodesic with different endpoints, proving the claim.

Lemma 5.2. The subgroup $\langle Q \cup R\rangle$ is relatively quasiconvex and its quasiconvexity constant is independent of the choice of $R$.

Proof. Let $f \in\langle Q \cup R\rangle$, and let $q$ be a geodesic in $\operatorname{Cayley}(G, X \cup \tilde{\mathscr{H}})$ from 1 to $f$. If $f \in Q \cup R$ then it is trivial that any vertex of $q$ is at most $\sigma$ apart from an element of $\langle Q \cup R\rangle$ with respect to the metric dist $_{X}$. Otherwise, let $p$ be the $\left(\lambda_{0}, 0\right)$-quasi-geodesic constructed during the proof of Lemma 5.1 from 1 to $f$. Notice that any vertex of $p$ is at most $\sigma$ apart from an element of $\langle Q \cup R\rangle$ with respect to the metric dist ${ }_{X}$. The BCP property implies that every vertex of $q$ is at most $\epsilon\left(\lambda_{0}, 0,0\right)$ apart from the set of vertices of $p$. It follows that any vertex of $q$ is at most $\left(\sigma+\epsilon\left(\lambda_{0}, 0,0\right)\right)$ apart from $\langle Q \cup R\rangle$. This shows that $\langle Q \cup R\rangle$ is relatively $\left(\sigma+\epsilon\left(\lambda_{0}, 0,0\right)\right)$-quasiconvex.

Lemma 5.3. Any parabolic element of the subgroup $\langle Q \cup R\rangle$ is either conjugate to an element of $Q$ or to an element of $R$ by an element of $\langle Q \cup R\rangle$.

Proof. If $f$ is a parabolic element of $G$, then its action on $\operatorname{Cayley}(G, X \cup \tilde{\mathcal{H}})$ fixes setwise a subset of diameter one. Indeed, if $f$ is a parabolic element, then there is an element $g \in G$ such that $g f g^{-1} \in H_{i}$ for some $i$. It follows that $f$ fixes setwise the left coset $g^{-1} H_{i}$ which has diameter one.

Let $f$ be an element of $Q * Q \cap R R$, and suppose that $f$ is not conjugate to an element of $Q$ or $R$. We claim that $f$ acts on a bi-infinite quasi-geodesic $\tilde{p}$ in $\operatorname{Cayley}(G, X \cup \tilde{\mathscr{H}})$ as a non-trivial translation, and hence the observation of the previous paragraph implies that $f$ is not a parabolic element.

Conjugate $f$, if necessary, and assume that its normal form,

$$
f=g_{1} h_{1} \ldots g_{k} h_{k},
$$

satisfies $g_{1} \neq 1$ and $h_{k} \neq 1$. Consider the path $o$ in $\operatorname{Cayley}(G, X \cup \tilde{\mathscr{H}})$

$$
o=u_{1} v_{1} \ldots u_{k} v_{k}
$$

from 1 to $f$, where each $u_{i}$ and $v_{i}$ are non-trivial geodesics in Cayley $(G, X \cup \tilde{\mathscr{H}})$, $\operatorname{Label}\left(u_{i}\right)$ represents $g_{i}$, and Label $\left(v_{i}\right)$ represents $h_{i}$. Let $\tilde{o}$ the bi-infinite path

$$
\tilde{o}=\ldots f^{-3}(o) f^{-2}(o) f^{-1}(o) o f(o) f^{2}(o) f^{3}(o) \ldots,
$$


and let $\tilde{p}$ the path obtained by replacing each $\mathscr{H}$-component of $\tilde{o}$ by a single edge. The argument of Lemma 5.1 shows that the subpath of $\tilde{p}$ induced by the subpath

$$
f^{-k}(o) f^{-k+1}(o) \ldots f^{-1}(o) o f(o) \ldots f^{k-1}(o) f^{k}(o)
$$

of $\tilde{o}$ is a $\left(\lambda_{0}, 0\right)$-quasi-geodesic for any integer $k>0$. It follows that $\tilde{p}$ is a bi-infinite $\left(\lambda_{0}, 0\right)$-quasi-geodesic, and that the (image in $G$ of the) element $f$ acts as a nontrivial translation on this bi-infinite quasi-geodesic.

Lemma 5.4. Any parabolic subgroup of $\langle Q \cup R\rangle$ is conjugate either to a subgroup of $Q$ or to a subgroup of $R$ by an element of $\langle Q \cup R\rangle$.

In particular, if $\left\{K_{1}, \ldots, K_{l}\right\}$ is the collection of maximal parabolic subgroups of $Q$ up to conjugacy in $Q$. Then the collection of maximal parabolic subgroups of $\langle Q \cup R\rangle$ up to conjugacy in $\langle Q \cup R\rangle$ is

(1) $\left\{R, K_{1}, \ldots, K_{l}\right\}$ if $Q \cap P$ is trivial;

(2) $\left\{R, K_{2}, \ldots, K_{l}\right\}$ if $Q \cap P$ and $K_{1}$ are conjugate in $Q$.

Proof. Assume that $Q \cap R$ is a proper subgroup of $R$ and $Q$; otherwise there is nothing to prove.

An argument using normal forms shows that if $J$ is a subgroup of $Q * Q \cap R R$ that cannot be conjugated into $Q$ or $R$, then $J$ contains an element that cannot be conjugated into $Q$ or $R$. By Lemma 5.3, any parabolic subgroup of $Q * Q \cap R R$ is conjugate to a parabolic subgroup of $Q$ or $R$.

The second statement of the lemma follows from the following observations. Since parabolic subgroups of $Q * Q \cap R R$ can be conjugated into subgroups of $Q$ or $R$, any maximal parabolic subgroup of $Q * Q \cap R R$ is conjugate to a subgroup in the collection $\left\{R, K_{1}, K_{2}, \ldots, K_{l}\right\}$. Since $K_{i}$ and $K_{j}$ are not conjugate in $Q$ for $i \neq j$, they are not conjugate in $Q * Q \cap R R$. Since $Q \cap R$ is a proper subgroup of $R$, no $K_{i}$ is conjugate to $R$ by an element $Q * Q \cap R R$.

5.2. Proof of Theorem 1.2. Conjugate the subgroups $Q_{1}$ and $Q_{2}$ if necessary and assume that $P=H$ for some $H \in \mathscr{H}$. A lower bound for the constant $C$ is given by (10) below. The proof is completely analogous to the proof of Theorem 1.1. Let $\sigma$ be a common quasiconvexity constant for $Q_{1}$ and $Q_{2}$.

Lemma 5.5. The natural homomorphism $Q_{1} *_{R} h Q_{2} h^{-1} \rightarrow G$ is injective.

Proof. Let $f$ be an element of $Q_{1} *_{R} h Q_{2} h^{-1}$ with normal form

$$
f=g_{1} g_{2}^{h} \ldots g_{2 k-1} g_{2 k}^{h},
$$

where $g_{2 i+1} \in Q_{1} \backslash R$ for $1 \leq i \leq k, g_{2 i} \in Q_{2} \backslash R$ for $1 \leq i<k$, either $g_{1}=1$ or $g_{1} \in Q_{1} \backslash R$, and either $g_{2 k}=1$ or $g_{2 k} \in Q_{2} \backslash R$. Consider the path $o$ in 
$\operatorname{Cayley}(G, X \cup \tilde{\mathscr{H}})$ from 1 to $f$ given by

$$
o=u_{1} v_{1} u_{2} v_{2} \ldots u_{2 k-1} v_{2 k-1} u_{2 k} v_{2 k}
$$

where $u_{i}, v_{2 i}$ and $v_{2 i+1}$ are geodesic paths in $\operatorname{Cayley}(G, X \cup \tilde{\mathscr{H}})$ such that $\operatorname{Label}\left(u_{i}\right)$ represents $g_{i}, \operatorname{Label}\left(v_{2 i-1}\right)$ represents $h$, and Label $\left(v_{2 i}\right)$ represents $h^{-1}$.

Claim 1. Let $t_{i}$ be the $\mathscr{H}$-component of $o$ that contains the subpath $v_{i}$. Then the $\mathscr{H}$-components $t_{i}$ and $t_{i+1}$ are not connected.

If $t_{2 i-1}$ and $t_{2 i}$ are connected $\mathscr{H}$-components, then $g_{2 i} \in R$, which contradicts the assumptions on (9). Analogously $t_{2 i}$ and $t_{2 i+1}$ are not connected $\mathscr{H}$-components.

Claim 2. $\operatorname{dist}_{X}\left(\left(t_{i}\right)_{-},\left(t_{i}\right)_{+}\right)>\eta$ for each $i$, where $\eta$ is the constant from Proposition 3.1 .

Fix an odd value of $i$, and let $x_{1}=\left(u_{i}\right)_{-}, x_{2}=\left(t_{i}\right)_{-}, x_{3}=\left(u_{i}\right)_{+}, x_{4}=\left(u_{i+1}\right)_{-}$, $x_{5}=t_{+}$and $x_{6}=\left(u_{i+1}\right)_{+}$. The argument used to prove Claim 2 of Lemma 5.1 shows that there are elements $z_{1}$ in the left coset $x_{3} R$, and $z_{2}$ in the left coset $x_{4} R$ such that

$$
\operatorname{dist}_{X}\left(x_{2}, z_{1}\right) \leq M\left(H, Q_{1}, \sigma\right)
$$

and

$$
\operatorname{dist}_{X}\left(x_{5}, z_{2}\right) \leq M\left(H, Q_{2}, \sigma\right),
$$

where $M\left(H, Q_{i}, \sigma\right)$ is the constant provided by Lemma 4.2 for the subgroups $H$ and $Q_{i}$, the constant $\sigma$, and the proper metric $d_{X}$. It follows that

$$
z_{1}^{-1} z_{2}=\left(z_{1}^{-1} x_{3}\right)\left(x_{3}^{-1} x_{4}\right)\left(x_{4}^{-1} z_{2}\right) \in R h R,
$$

and hence $\operatorname{dist}_{X}\left(z_{1}, z_{2}\right) \geq C$. Now the triangle inequality and our choice of $C$ implies that

$$
\begin{aligned}
\operatorname{dist}_{X}\left(x_{2}, x_{5}\right) & \geq \operatorname{dist}_{X}\left(z_{1}, z_{2}\right)-\operatorname{dist}_{X}\left(x_{2}, z_{1}\right)-\operatorname{dist}_{X}\left(x_{5}, z_{2}\right) \\
& \geq C-M\left(H, Q_{1}, \sigma\right)-M\left(H, Q_{2}, \sigma\right) \\
& \geq \eta
\end{aligned}
$$

where $\eta$ is the constant from Proposition 3.1 The case for an even value of $i$ is similar, the only difference is that $z_{1}^{-1} z_{2} \in R h^{-1} R$, which also implies $\operatorname{dist}_{X}\left(z_{1}, z_{2}\right) \geq C$.

Claim 3. Let $p$ be the path obtained by replacing each $\mathscr{H}$-component $v_{i}$ of $o$ by a single edge $s_{i}$. The above claims and Proposition 3.1 imply that $p$ is $\left(\lambda_{0}, 0\right)$ quasi-geodesic with different endpoints. In particular the image of $f$ by the map $Q_{1} *_{R} h Q_{2} h^{-1} \longrightarrow G$ is not trivial.

Remark 5.6. If $p$ is the path from 1 to an element $f$ of $\left\langle Q_{1}, h Q_{2} h^{-1}\right\rangle \backslash Q_{1} \cup Q_{2}$ constructed in the proof, then $p$ has at least two different $\mathscr{H}$-components $s_{1}$ and $s_{2}$ of $X$-length at least $\eta$, namely the ones induced by an element of $h Q_{2} h^{-1}$ in the normal 
form of $f$. Since $\eta$ is larger than $\epsilon\left(\lambda_{0}, 0,0\right)$ (see (6)), the BCP property implies that any geodesic from 1 to $f$ has at least two different $\mathscr{H}$-components. In particular, the element $f$ does not belong to a subgroup $H \in \mathscr{H}$.

Therefore, if $Q_{1} \cap H=Q_{2} \cap H$ is the trivial subgroup, then $\left\langle Q_{1} \cup h Q_{2} h^{-1}\right\rangle \cap H$ is trivial.

Similar arguments to the proofs of Lemmas 5.3 and 5.2 show the following.

Lemma 5.7. The subgroup $\left\langle Q_{1} \cup h Q_{2} h^{-1}\right\rangle$ is relatively quasiconvex.

Lemma 5.8. Every parabolic subgroup of $\left\langle Q_{1} \cup h Q_{2} h^{-1}\right\rangle$ is conjugate to a parabolic subgroup of $Q_{1}$ or $Q_{2}$ by an element of $\left\langle Q_{1} \cup h Q_{2} h^{-1}\right\rangle$.

In particular, if $\mathcal{K}_{i}$ is the collection of maximal parabolic subgroups of $Q_{i}$ up to conjugacy in $Q_{i}$ for $i=1,2$, then every maximal parabolic subgroup of $\left\langle Q_{1} \cup h Q_{2} h^{-1}\right\rangle$ is conjugate to a subgroup in $\mathcal{K}_{1} \cup \mathcal{K}_{2}$ by an elementof $\left\langle Q_{1} \cup h Q_{2} h^{-1}\right\rangle$.

\subsection{Proof of Corollary 1.7}

Lemma 5.9. Suppose that $A$ is an abelian group with a finite generating set $Y, B$ is a subgroup of $A$, and $h \in A$ such that

$$
\operatorname{rank}_{\mathbb{Z}}(B)<\operatorname{rank}_{\mathbb{Z}}(\langle B \cup\{h\}\rangle) .
$$

Then there is a constant $\lambda=\lambda(B, h, Y)$ such that $|g|_{Y} \geq \lambda|j|$ for any $j \in \mathbb{Z}$ and $g \in h^{j} B$.

Proof. Since $h$ has infinite order, one can regard $A$ as the direct product $A_{1} \oplus\left\langle h_{1}\right\rangle$, where $h \in\left\langle h_{1}\right\rangle$ and $B \subset A_{1}$. Suppose that $Y$ contains $h_{1}$ and $Y \backslash\left\{h_{1}\right\}$ generates $A_{1}$. Then $|g|_{Y} \geq\left|h^{j}\right|_{Y} \geq|j|$ for any $g \in h^{j} B$. Since the word metrics associated to different finite generating sets are Lipschitz equivalent the result follows.

Corollary 1.7 follows from the following proposition.

Proposition 5.10. Let $G$ be hyperbolic relative to a collection of free abelian subgroups $H, Q$ a relatively quasiconvex subgroup, and $P$ a maximal parabolic subgroup of $G$. If

$$
\operatorname{rank}_{\mathbb{Z}}(Q \cap P)<\operatorname{rank}_{\mathbb{Z}}(P),
$$

then there is an element $h \in P$ with the following property. For any positive integer $k$, there exist integers $n_{1}, \ldots, n_{k}$ such that

$$
\left\langle\bigcup_{i=1}^{k} h^{n_{i}} Q h^{-n_{i}}\right\rangle \cong \Delta_{k}(Q, Q \cap H) .
$$

Moreover, the above subgroup is relatively quasiconvex. 
Proof. Let $G$ be hyperbolic relative to a collection of abelian subgroups $\mathscr{H}, Q$ a relatively quasiconvex subgroup, and $H \in \mathscr{H}$ such that

$$
\operatorname{rank}_{\mathbb{Z}}(Q \cap H)<\operatorname{rank}_{\mathbb{Z}}(H) .
$$

Let $h \in H$ be any element such that

$$
\operatorname{rank}_{\mathbb{Z}}(Q \cap H)<\operatorname{rank}_{\mathbb{Z}}(\langle(Q \cap H) \cup\{h\}\rangle),
$$

and let $Y$ be a finite generating set of $H$. By Lemma 5.9 there is a constant $\lambda>0$ such that

$$
|g|_{Y} \geq \lambda|j|
$$

for any integer $j$ and any element $g$ in the left coset $h^{j}(Q \cap H)$.

By induction on $k$, we prove the existence of integers $n_{1}, \ldots, n_{k}$ such that the subgroup $\left\langle\bigcup_{i=1}^{k} h^{n_{i}} Q h^{-n_{i}}\right\rangle$ is quasiconvex, isomorphic to $\Delta_{k}(Q, Q \cap H)$, and $Q \cap H=\left\langle\bigcup_{i=1}^{k} h^{n_{i}} Q h^{-n_{i}}\right\rangle \cap H$.

The case $k=1$ is trivial taking $n_{1}=0$. Suppose that $R_{k-1}=\left\langle\bigcup_{i=1}^{k-1} f^{n_{i}} Q f^{-n_{i}}\right\rangle$ is a quasiconvex subgroup isomorphic to $\Delta_{k-1}(Q, Q \cap H)$, and $R_{k-1} \cap H=Q \cap H$. Let $C=C\left(R_{k-1}, Q, H\right)$ be the constant provided by Theorem 1.2, and let $n_{k}$ be any integer such that

$$
\max \left\{|g|_{Y} \mid g \in H \text { with }|g|_{X}<C\right\}<\lambda n_{k} .
$$

Now we show that the quasiconvex subgroups $R_{k-1}$ and $Q$, the maximal parabolic subgroup $H$, and the element $h^{n_{k+1}} \in H$ satisfy the hypothesis of Theorem 1.2: first, since $H$ is abelian, $h^{n_{k}}(Q \cap H) h^{-n_{k}}=Q \cap H$; second, if $g$ is an element of the left coset $h^{n_{k+1}}(Q \cap H)$, then (11) and (12) imply that $|g|_{X} \geq C$.

Therefore Theorem 1.2 implies that the subgroup $R_{k}=\left\langle R_{k-1}, h^{n_{k}} Q h^{-n_{k}}\right\rangle$ is isomorphic to $R_{k-1} * Q \cap H Q \cong \Delta_{k}(Q, Q \cap H)$ and is quasiconvex. We claim that $Q \cap H=R_{k} \cap H$.

If $Q \cap H$ is not trivial, then Lemma 5.8 applied to $Q$ and $R_{k-1}$ implies that $Q \cap H$ is a maximal parabolic subgroup of $R_{k}$. Since $R_{k} \cap H$ is a maximal parabolic subgroup of $R_{k}$ containing $Q \cap H$, if follows that $Q \cap H=R_{k} \cap H$. If $Q \cap H$ is trivial, then Remark 5.6 implies that $R_{k} \cap H$ is trivial.

5.4. Proof of Corollary 1.8. Corollary 1.8 follows from the following proposition.

Proposition 5.11. Let $G$ be hyperbolic relative to a collection of free abelian subgroups $\mathcal{H}$, and let $Q$ be a relatively quasiconvex subgroup. Then there exists a fully quasiconvex subgroup $R$ which has the tree of groups decomposition described in Figure 8 , where $\left\{K_{1}, \ldots, K_{n}\right\}$ is a collection of representatives of the maximal parabolic subgroups of $Q$ and each $A_{i}$ is a finite index subgroup of a maximal parabolic subgroup of $G$. 


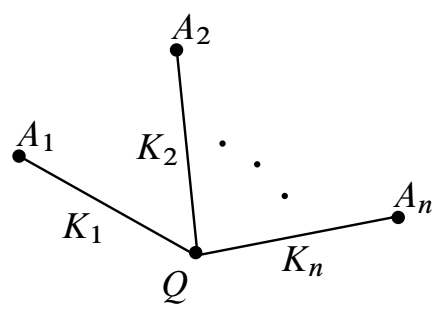

Figure 8. Tree of groups decomposition of the fully quasiconvex subgroup $R$ of Corollary 1.8 .

Proof. By Proposition 1.5, a collection of representatives of the infinite maximal parabolic subgroups of $Q$ up to conjugacy in $Q$ is finite, say $K_{1}, \ldots, K_{n}$. The desired group is obtained after an $n$-step process which produces a sequence $\left\{Q_{j}\right\}_{j=0}^{n}$ of quasiconvex subgroups of $G$, where $Q_{0}=Q$ and $Q_{n}=R$ is a fully quasiconvex subgroup. For $1 \leq j \leq n$, the group $Q_{j}$ has the graph of groups decomposition described in Figure 9, where $\left\{A_{1}, \ldots, A_{j}, K_{j+1}, \ldots, K_{n}\right\}$ is the collection of all maximal parabolic subgroups of $Q_{j}$ up to conjugation in $Q_{j}$, and $A_{i}$ is a finite index subgroup of a maximal parabolic subgroup of $G$ for each $i \leq j$.

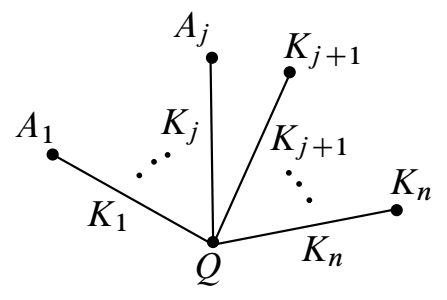

Figure 9. The graph of groups decomposition of the subgroup $Q_{j}$ in the proof of Corollary 1.8 .

Now we explain how to obtain $Q_{i+1}$ from $Q_{i}$ when $i<n$. Let $P$ be the maximal parabolic subgroup of $G$ containing $K_{i+1}$, and let $Y$ be a finite generating set of $P$. Let $C=C\left(Q_{i}, P\right) \geq 0$ the constant provided by Theorem 1.1, and define

$$
D=\max \left\{|g|_{Y} \mid g \in P \text { with }|g|_{X}<C\right\} .
$$

Since $P$ is a finitely generated abelian group, there is a finite index subgroup $A_{i+1}$ of $P$ containing $K_{i+1}$ such that $|g|_{Y} \geq D$ for any $g \in A \backslash K$. In particular $|g|_{X} \geq C$ for any $g \in A_{i+1} \backslash K_{i+1}$, and hence Theorem 1.1 and Lemma 5.4 imply that the subgroup $Q_{i+1}=\left\langle Q_{i} \cup A_{i+1}\right\rangle$ of $G$ is isomorphic to $Q_{i} *_{K_{i+1}} A_{i+1}$, is relatively quasiconvex, and $\left\{A_{1}, \ldots, A_{j+1}, K_{j+2}, \ldots, K_{n}\right\}$ is the collection of all maximal parabolic subgroups of $Q_{i+1}$ up to conjugation in $Q_{i+1}$. 


\section{Future directions}

6.1. Amalgamation along hyperbolic subgroups. The main results of this paper address Problem 1, stated in the introduction, in the case that $K$ is a maximal parabolic subgroup of $Q_{1}$ or $Q_{2}$. The case that $K$ consists only of hyperbolic elements remains to be considered.

An analogous version of Problem 1 for HNN-extensions is of interest.

Problem 2. Let $R_{1}$ and $R_{2}$ are subgroups of a relatively quasiconvex subgroup $Q$ of $G$. Investigate conditions that guarantee the existence of a group homomorphism $\varphi: R_{1} \rightarrow R_{2}$ and an injective homomorphism

$$
Q *_{\varphi} \rightarrow G
$$

with image a quasiconvex subgroup. Here $Q *{ }_{\varphi}$ represents the $\mathrm{HNN}$-extension

$$
\left\langle Q, t \mid t r t^{-1}=\varphi(r), r \in R_{1}\right\rangle .
$$

6.2. Surface subgroups in (relatively) hyperbolic groups. Finding subgroups isomorphic to fundamental groups of hyperbolic closed surfaces in (relatively) hyperbolic groups has been an theme in Geometric Group Theory [8], [27]. In this context, D. Calegari has shown that if a group $G$ decomposes as a graph of free groups amalgamated over cyclic subgroups and the second rational homology is not trivial, then $G$ contains a surface subgroup [11]. D. Cooper, D. Long and A. Reid have produced closed surface subgroups in fundamental groups of complete hyperbolic 3-manifolds with cusps [13], [12]. In [10], N. Brady, M. Forester and the author have shown that a class of word hyperbolic groups of the form $F_{k} * \mathbb{Z} F_{l}$, where $F_{k}$ and $F_{l}$ are free groups of rank $k$ and $l$ respectively, have surfaces subgroups. The techniques used in [10] resemble Cooper-Long-Reid ideas of doubling surfaces with one boundary component through a combination theorem.

Problem 3. For which other classes of word hyperbolic groups can these ideas produce surface subgroups?

D. Cooper and D. Long have produced surface subgroups in Dehn fillings of hyperbolic manifolds [13]. In the context of hyperbolic Dehn fillings on relatively hyperbolic groups [19], [26] we set

Problem 4. Explore the existence of surface subgroups in one-ended word hyperbolic groups arising as hyperbolic Dehn fillings of relatively hyperbolic groups. 


\section{References}

[1] I. Agol, D. Groves, J. Manning, Residual finiteness, QCERF, and fillings of hyperbolic groups. Geom. Topol. 13 (2009), 1043-1073.

[2] E. Alibegović, A combination theorem for relatively hyperbolic groups. Bull. London Math. Soc. 37 (2005), 459-466. Zbl 1074.57001 MR 2131400

[3] J. W. Anderson, J. Aramayona, and K. J. Shackleton, An obstruction to the strong relative hyperbolicity of a group. J. Group Theory 10 (2007), 749-756. Zbl 05222399 MR 2364824

[4] G. N. Arzhantseva, On quasiconvex subgroups of word hyperbolic groups. Geom. Dedicata 87 (2001), 191-208. Zbl 0994.20036 MR 1866849

[5] G. Arzhantseva and A. Minasyan, Relatively hyperbolic groups are $C^{*}$-simple. J. Funct. Anal. 243 (2007), 345-351. Zbl 1115.20034 MR 2291441

[6] M. Baker and D. Cooper, A combination theorem for convex hyperbolic manifolds, with applications to surfaces in 3-manifolds. J. Topology 1 (2008), 603-642. Zbl 1151.57014 MR 2417445

[7] J. Behrstock, C. Drutu, and L. Mosher, Thick metric spaces, relative hyperbolicity, and quasi-isometric rigidity. Preprint 2005. arXiv:math/0512592

[8] M. Bestvina, Questions in geometric group theory. Preprint 2004.

http://www.math.utah.edu/ bestvina/eprints/questions-updated.pdf

[9] B. H. Bowditch, Relatively hyperbolic groups. Preprint 1999. http://www.warwick.ac.uk/ masgak/papers/bhb-relhyp.pdf

[10] N. Brady, M. Forester, and E. Martinez-Pedroza, Surface subgroups of hyperbolization of groups. In preparation.

[11] D. Calegari, Surface subgroups from homology. Geom. Topol. 12 (2008), 1995-2007. Zbl 05344743 MR 2431013

[12] D. Cooper, D. D. Long, and A. W. Reid, Essential closed surfaces in bounded 3-manifolds. J. Amer. Math. Soc. 10 (1997), 553-563. Zbl 0896.57009 MR 1431827

[13] D. Cooper and D. D. Long, Some surface subgroups survive surgery. Geom. Topol. 5 (2001), 347-367. Zbl 1009.57017 MR 1825666

[14] F. Dahmani, Combination of convergence groups. Geom. Topol. 7 (2003), 933-963. Zbl 1037.20042 MR 2026551

[15] C. Druţu and M. Sapir, Tree-graded spaces and asymptotic cones of groups. Topology 44 (2005), 959-1058. Zbl 1101.20025 MR 2153979

[16] B. Farb, Relatively hyperbolic groups. Geom. Funct. Anal. 8(1998), 810-840. Zbl 0985.20027 MR 1650094

[17] R. Gitik, Ping-pong on negatively curved groups. J. Algebra 217 (1999), 65-72. Zbl 0936.20019 MR 1700476

[18] M. Gromov, Hyperbolic groups. In Essays in group theory, Math. Sci. Res. Inst. Publ. 8, Springer-Verlag, New York 1987, 75-263. Zbl 0634.20015 MR 0919829 
[19] D. Groves and J. F. Manning, Dehn filling in relatively hyperbolic groups. Israel J. Math. 168 (2008), 317-429. Zbl 05508729 MR 2448064

[20] G. C. Hruska, Relative hyperbolicity and relative quasiconvexity for countable groups. Preprint 2008. arXiv:0801.4596

[21] C. J. Leininger and A. W. Reid, A combination theorem for Veech subgroups of the mapping class group. Geom. Funct. Anal. 16 (2006), 403-436. Zbl 1099.57002 MR 2231468

[22] J. F. Manning and E. Martinez-Pedroza, Separation of relatively quasiconvex subgroups. Preprint 2008. arXiv:0811.4001

[23] B. Maskit, Kleinian groups. Grundlehren Math. Wiss. 287, Springer-Verlag, Berlin 1988. Zbl 0627.30039 MR 0959135

[24] H. A. Masur and Y. N. Minsky, Geometry of the complex of curves I: hyperbolicity. Hyperbolicity. Invent. Math. 138 (1999), 103-149. Zbl 0941.32012 MR 1714338

[25] D. V. Osin, Relatively hyperbolic groups: intrinsic geometry, algebraic properties, and algorithmic problems. Mem. Amer. Math. Soc. 179 (2006), no. 843. Zbl 1093.20025 MR 2182268

[26] D. V. Osin, Peripheral fillings of relatively hyperbolic groups. Invent. Math. 167 (2007), 295-326. Zbl 1116.20031 MR 2270456

[27] M. V. Sapir, Some group theory problems. Internat. J. Algebra Comput. 17 (2007), 1189-1214. Zbl 05275105 MR 2355692

[28] J. Stallings, A finitely presented group whose 3-dimensional integral homology is not finitely generated. Amer. J. Math. 85 (1963), 541-543. Zbl 0122.27301 MR 0158917

[29] A. Szczepański, Relatively hyperbolic groups. Michigan Math. J. 45 (1998), 611-618. Zbl 0962.20031 MR 1653287

[30] A. Yaman, A topological characterisation of relatively hyperbolic groups. J. Reine Angew. Math. 566 (2004), 41-89. Zbl 1043.20020 MR 2039323

Received October 15, 2007; revised August 29, 2008

E. Martínez-Pedroza, Department of Mathematics and Statistics, McMaster University,

Hamilton, Ontario L8S 4K1, Canada

E-mail: emartinez@math.mcmaster.ca 